\title{
RETRIEVING CONVEX BODIES FROM RESTRICTED COVARIOGRAM FUNCTIONS
}

\author{
GENNADIY AVERKOV, ${ }^{*}$ University of Magdeburg \\ GABRIELE BIANCHI, ${ }^{* *}$ Università di Firenze
}

\begin{abstract}
The covariogram $g_{K}(x)$ of a convex body $K \subseteq \mathbb{E}^{d}$ is the function which associates to each $x \in \mathbb{E}^{d}$ the volume of the intersection of $K$ with $K+x$, where $\mathbb{E}^{d}$ denotes the Euclidean $d$-dimensional space. Matheron (1986) asked whether $g_{K}$ determines $K$, up to translations and reflections in a point. Positive answers to Matheron's question have been obtained for large classes of planar convex bodies, while for $d \geq 3$ there are both positive and negative results. One of the purposes of this paper is to sharpen some of the known results on Matheron's conjecture indicating how much of the covariogram information is needed to get the uniqueness of determination. We indicate some subsets of the support of the covariogram, with arbitrarily small Lebesgue measure, such that the covariogram, restricted to those subsets, identifies certain geometric properties of the body. These results are more precise in the planar case, but some of them, both positive and negative ones, are proved for bodies of any dimension. Moreover some results regard most convex bodies, in the Baire category sense. Another purpose is to extend the class of convex bodies for which Matheron's conjecture is confirmed by including all planar convex bodies possessing two nondegenerate boundary arcs being reflections of each other.
\end{abstract}

Keywords: Convex body; convex polytope; covariogram; genericity result; geometric tomography; set covariance; quasicrystal

2000 Mathematics Subject Classification: Primary 52A20

Secondary 52A22; 52A38; 60D05

\section{Introduction}

Let $K$ be a convex body in $\mathbb{E}^{d}$. The covariogram $g_{K}$ of $K$ is the function

$$
g_{K}(x)=V(K \cap(K+x)),
$$

where $x \in \mathbb{E}^{d}$ and $V$ denotes the volume in $\mathbb{E}^{d}$. This functional, which was introduced by Matheron in his book [20] on random sets, is also sometimes called the set covariance, and it coincides with the autocorrelation of the characteristic function $\mathbf{1}_{\{K\}}$ of $K$, i.e.

$$
g_{K}=\mathbf{1}_{\{K\}} * \mathbf{1}_{\{-K\}} .
$$

The covariogram $g_{K}$ is clearly unchanged by a translation or a reflection of $K$. (The term reflection will always mean reflection in a point.) Matheron [21] and, independently, Adler and Pyke [2] asked the following question.

Received 16 October 2006; revision received 5 April 2007.

* Postal address: Faculty of Mathematics, University of Magdeburg, Universitätsplatz 2, D-39106 Magdeburg,

Germany. Email address: gennadiy.averkov@googlemail.com

** Postal address: Department of Mathematics, Università di Firenze, Viale Morgagni 67a, 50134 Firenze, Italy.

Email address: gabriele.bianchi@unifi.it 
Covariogram problem. Does the covariogram determine a convex body, among all convex bodies, up to translations and reflections?

Matheron conjectured a positive answer for the case in which $d=2$, but this conjecture has not been completely settled.

Matheron [20, p. 86] observed that, for $u \in \mathbb{S}^{d-1}$ and for all $r>0$, the derivatives $\partial g_{K}(r u) / \partial r$ give the distribution of the lengths of the chords of $K$ parallel to $u$. Such information is common in stereology, statistical shape recognition, and image analysis, when properties of an unknown body are to be inferred from chord length measurements; see [25], [10], and [29], for example. Blaschke (cf. [24]) asked whether the distribution of the lengths of chords (in all directions) of a convex body characterizes the body, up to rigid motions, but Mallows and Clark [19] proved that this is false even for convex polygons. In fact (see [23]) the covariogram problem is equivalent to the problem of determining a convex body from all its separate chord length distributions, one for each direction $u \in \mathbb{S}^{d-1}$.

Adler and Pyke [1], [2] asked Matheron's question in probabilistic terms. Does the distribution of the difference $X-Y$ of independent random variables $X$ and $Y$ uniformly distributed over $K$ determine $K$, up to translations and reflections? Since the convolution in (1) is, up to a multiplicative factor, the probability density of $X-Y$, this problem is equivalent to the covariogram one.

Matheron's problem is also relevant in X-ray crystallography, where the atomic structure of a crystal (or quasicrystal) is to be found from diffraction images. A convenient way of describing many important examples of quasicrystals is via the cut and project scheme. Here, to the atomic structure, represented by a discrete set $S$ contained in a space $E$, is associated a lattice $N$ in a higher-dimensional space $E \times E^{\prime}$ and a window $W \subset E^{\prime}$ (which in many cases is a convex set). In this setting, $S$ coincides with the projection on $E$ of the points of the lattice $N$ which belong to $E \times W$. In many examples the lattice $N$ can be determined by the diffraction image. To determine $S$ it is however necessary to know $W$ : the covariogram problem enters at this point, since the covariogram of $W$ can be obtained by the diffraction image; see [4].

Enns and Ehlers [11], [12], [13] express, in terms of the covariogram, the distributions of random line segments in a convex body, under different types of randomness with which they are generated. The monograph [15] contains an extensive discussion of retrieval problems for convex bodies, while the survey [30] deals with algorithmic aspects of reconstruction problems in convex geometry.

The first contribution to Matheron's question was made in 1993 by Nagel [23], who gave a positive answer when $K$ is a planar convex polygon; see also Schmitt [25]. Matheron's conjecture is still unsettled for general planar convex bodies, but it has been confirmed for $\mathcal{C}^{2}$ convex bodies, nonstrictly convex bodies, and convex bodies that are not $\mathcal{C}^{1}$; see [6]. It has recently been shown that every convex polytope in $\mathbb{E}^{3}$ is determined by its covariogram, up to translations and reflections; see [8]. For $d \geq 4$ there exist examples of convex polytopes that are not determined by their covariogram; see [6]. However, [16, p. 87] proves that, if $P$ is a $d$-dimensional simplicial convex polytope in general relative position with respect to $-P$, then the determination by the covariogram data is unique for every $d \geq 2$ (see Section 2 for all unexplained definitions). Bianchi [7] discussed various open retrieval problems related to the covariogram.

One of the purposes of this paper is to sharpen some of the known results on Matheron's conjecture, indicating how much of the covariogram information is needed to get the uniqueness of determination. We indicate some subsets of the support of the covariogram, with arbitrarily small Lebesgue measure, such that the covariogram, restricted to those subsets, identifies certain 
geometric properties of the body. These results are more precise in the planar case, but some of them, both positive and negative ones, are proved for bodies of any dimension. Moreover some results regard most convex bodies, in the Baire category sense. Another purpose is to extend the class of convex bodies for which Matheron's conjecture is confirmed by including all planar convex bodies possessing two nondegenerate boundary arcs being reflections of each other.

Given two convex bodies $K$ and $H$ in $\mathbb{E}^{d}$ and a closed set $X \subseteq \mathbb{E}^{d}$, we introduce the following property involving $K, H$, and $X$ (GC stands for 'covariogram coincidence', where 'covariogram' is traditionally denoted by the letter $\mathrm{G})$ :

$\mathrm{GC}(X)$ the equality $g_{K}(x)=g_{H}(x)$ holds for all $x$ in some neighborhood of $X$.

Theorem 1, below, presents two choices of the set $X$ for which $\mathrm{GC}(X)$ implies the coincidence of $K$ and $H$ up to translations and reflections, under the assumption $K \in \mathcal{C}_{+}^{2}$. Before stating the theorem we need to introduce the notion of local symmetry and give some related explanations.

A pair of closed boundary arcs of a planar convex body $K$ is said to be a local symmetry of $K$ if they are reflections of each other in a point, have disjoint and nonempty relative interiors, and they are not properly contained in a pair of boundary arcs with the same properties. A planar convex body $K$ is called locally symmetric if it possesses a local symmetry. Planar convex bodies without local symmetries are called globally nonsymmetric. It is known that the support of $g_{K}$ is the difference body of $K, D K=\{x-y: x, y \in K\}$, and that $D K$ is $o$-symmetric. If $A^{+}$and $A^{-}$are arcs of bd $K$ (where bd stands for boundary) which compose a local symmetry, then the set $2\left(A^{+} \cup A^{-}\right)$, translated in such a way to be $o$-symmetric, is the union of two $\operatorname{arcs} A$ and $-A$ of $\operatorname{bd} D K$. We say that these $\operatorname{arcs}$ of bd $D K$ correspond to the local symmetry $A^{+}, A^{-}$; see Figure 1.

A convex body $K$ is said to belong to the class $\mathcal{C}_{+}^{2}$ if its boundary is a two-times continuously differentiable manifold and all its principal curvatures are nonzero (for detailed information, see [26, Section 2.5]).

Theorem 1. Let $K$ and $H$ be planar convex bodies and let $K$ be $\mathcal{C}_{+}^{2}$ regular. Let $\left\{ \pm A_{n}\right\}_{n \in \mathcal{N}}$ be the collection of all the arcs of $\mathrm{bd} D K$ which correspond to local symmetries of $K$, for a suitable $\mathcal{N} \subset \mathbb{N}$. By $x_{n}$ we denote the midpoint of the segment joining the endpoints of the arc $A_{n}$. Let $X_{0}:=\left\{ \pm x_{n}: n \in \mathcal{N}\right\}$ and let $X=X_{0} \cup \operatorname{bd} D K$ or $X=X_{0} \cup\{o\}$; see Figure 1. Then $\mathrm{GC}(X)$ implies the coincidence of $K$ and $H$, up to translations and reflections.

(a)

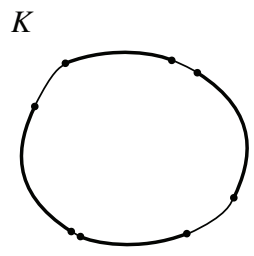

(b)

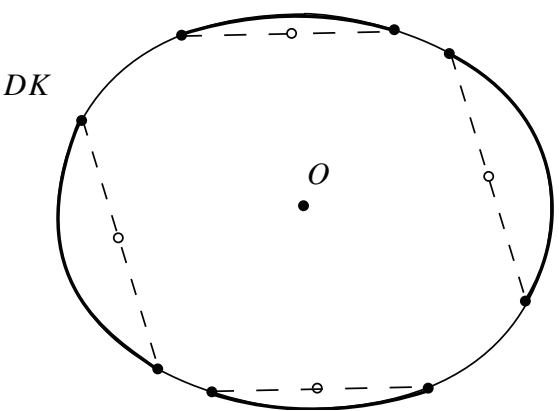

Figure 1: Part (a) shows the body $K$ with two local symmetries plotted in bold. Part (b) shows arcs $\pm A_{n}$ of $D K$, corresponding to local symmetries of $K$, plotted in bold; points of $X_{0}$ are depicted by open circles. 
We remark that the two choices of $X$ defined in the statement of Theorem 1 are in some sense minimal for the assertion of the theorem to hold (for further details see Remark 1, below). Moreover, the set $X_{0}$ depends only on $g_{K}$ and not on $K$, in the sense that if $H$ and $K$ satisfy $\mathrm{GC}(\mathrm{bd} D K)$ or $\mathrm{GC}(o)$, then the same set $X_{0}$ corresponds to $H$ and $K$. This is the content of the second part of Theorem 2, below.

The following corollary is a direct consequence of Theorem 1.

Corollary 1. Let $K$ and $H$ be planar convex bodies and let $K$ be $\mathcal{C}_{+}^{2}$ regular. Then the following statements hold.

(i) There exists a closed and at most countable subset $X$ of $D K$, with no accumulation points in int $D K$ (where int stands for interior) such that $\mathrm{GC}(X \cup\{o\})$ implies the coincidence of $K$ and $H$, up to translations and reflections. Furthermore, $X$ can be chosen to be lying on a strictly convex curve.

(ii) If $K$ is globally nonsymmetric and $X$ is either $\{o\}$ or $\operatorname{bd} D K$, then $\mathrm{GC}(X)$ implies the coincidence of $K$ and $H$, up to translations and reflections.

It is an open question whether Corollary 1(ii) holds for all strictly convex $K$ not necessarily $\mathcal{C}_{+}^{2}$. The positive answer to this question would imply the confirmation of Matheron's conjecture for all planar convex bodies.

The following theorem presents determination results involving locally symmetric and symmetric convex bodies.

Theorem 2. Let $K$ and $H$ be planar convex bodies with $K$ strictly convex. Then the following statements hold.

(i) If $K$ is o-symmetric and $A$ is a closed simple curve in int $D K$ bounding an open region in $D K$ that contains the origin, then the conditions $D K=D H$ and $\mathrm{GC}(A)$ imply the coincidence of $K$ and $H$, up to translations and reflections.

(ii) If $X$ is either $\{o\}$ or $\operatorname{bd} D K$, then $\mathrm{GC}(X)$ implies the coincidence of all local symmetries of $K$ and $H$, up to translations.

The statement of the following corollary follows directly from Theorem 2(ii) and [6, Theorem 1.1 and Proposition 1.4].

Corollary 2. Every locally symmetric convex body in $\mathbb{E}^{2}$ is determined uniquely, up to translations and reflections, by its covariogram function.

Let $\mathbb{B}^{d}$ denote the closed unit ball in $\mathbb{E}^{d}$ centered at the origin. Furthermore, we introduce the condition $\mathrm{GC}^{\prime}(X)$, a relaxation of $\mathrm{GC}(X)$, and the 'local coincidence' condition LC:

$\mathrm{GC}^{\prime}(X)$ the equality $g_{K}(x)=g_{H}(x)+c$ holds for all $x$ in some neighborhood of $X$ and a suitable constant $c \in \mathbb{R}$,

LC for every boundary point $p$ of $K$ there exists a boundary point $q$ of $H$ such that for some $\varepsilon>0$ the bodies $K \cap\left(p+\varepsilon \mathbb{B}^{d}\right)$ and $H \cap\left(q+\varepsilon \mathbb{B}^{d}\right)$ coincide, up to translations and reflections (the same statement also holds with the roles of $K$ and $H$ interchanged).

The relationship between the conditions $\mathrm{GC}(\{o\}), \mathrm{GC}^{\prime}(\{o\}), \mathrm{GC}(\mathrm{bd} D K)$, and $\mathrm{LC}$ is discussed in the following theorem. 
Theorem 3. Let $K$ and $H$ be convex bodies in $\mathbb{E}^{d}, d \geq 2$. Then the following statements hold.

(i) If $K$ is strictly convex, then LC implies $\mathrm{GC}(\mathrm{bd} D K)$.

(ii) If $d=2$ and $K$ is $\mathcal{C}_{+}^{2}$, then $\mathrm{LC}$ is equivalent to $\mathrm{GC}(\mathrm{bd} D K)$.

(iii) If $d=2$ and $K$ is strictly convex, then $\mathrm{GC}^{\prime}(\{o\})$ is equivalent to $\mathrm{GC}(\mathrm{bd} D K)$.

(iv) There exist planar convex bodies $K$ and $H$ belonging to the class $\mathcal{C}_{+}^{2}$ such that $\mathrm{GC}(\mathrm{bd} D K)$ holds, while $\mathrm{GC}(\{0\})$ does not.

(v) There exist $K$ and $H$, which are convex d-polytopes, such that the conditions $D K=D H$ and $\mathrm{GC}(\{o\})$ hold, while $\mathrm{GC}(\mathrm{bd} D K)$ does not.

It is an open problem whether, for $\mathcal{C}_{+}^{2}$ convex bodies in $\mathbb{E}^{d}, d \geq 3$, the condition $\operatorname{GC}(\operatorname{bd} D K)$ implies LC.

The space $\mathcal{K}^{d}$ endowed with the Hausdorff metric is locally compact and therefore a Baire space; see [17] and [26, p. 119]. Thus, we may speak about statements that hold for most convex bodies, i.e. for all convex bodies with at most a meager set of exceptions. We recall that a set is said to be meager if it is a countable union of nowhere dense sets and residual if it is a complement of a meager set. Trivially, a finite intersection of residuals is a again a residual. Furthermore, every set possessing a residual subset is also a residual.

Theorem 4. In the Baire category sense, for most convex bodies $K$ and all convex bodies $H$ in $\mathbb{E}^{d}$, the following statements hold.

(i) If $K$ and $H$ satisfy $\mathrm{GC}(\mathrm{bd} D K)$, then $K$ and $H$ coincide, up to translations and reflections.

(ii) If $d=2$ and $K$ and $H$ satisfy $\mathrm{GC}(\{o\})$, then $K$ and $H$ coincide, up to translations and reflections.

Furthermore, the above two statements cannot be extended to all pairs of convex bodies $K$ and $H$, since, for each $d \geq 2$, there exists bodies $K$ and $H$ in $\mathbb{E}^{d}$ not coinciding, up to translations and reflections, and such that $\mathrm{GC}(\mathrm{bd} D K \cup\{o\})$ holds. In addition, the bodies $K$ and $H$ satisfying the above conditions can be chosen belonging to the class $\mathcal{C}_{+}^{2}$.

It is an open question whether Theorem 4(ii) can be carried over to convex bodies of higher dimensions.

Theorem 4 is related to [16, Theorem 2] and [9, Theorem 6.2]. Theorem 2 of [16] states that most convex bodies $K$ in $\mathbb{E}^{d}$, for any $d \geq 3$, are determined by the combined knowledge of the width of $K$ in direction $u$ and of the $(d-1)$-dimensional volume of $K \mid u^{\perp}$ for each $u \in \mathbb{S}^{d-1}$. Here, $u^{\perp}$ denotes the orthogonal complement to $u$, and $K \mid u^{\perp}$ stands for the orthogonal projection of $K$ onto the hyperplane $u^{\perp}$. (See also [28], where 'local versions' of some results from [16] are obtained, as well as [15, Theorems 3.3.17 and 3.3.18].) The relation with the covariogram comes from the fact that knowing its support $D K$ is equivalent to knowing the width of $K$ in all directions, and that the knowledge of $g_{K}$ in a neighborhood of $o$ gives the volumes of all $(d-1)$-dimensional projections of $K$. This follows from the formula

$$
\left.\frac{\partial^{+} g_{K}(r u)}{\partial r}\right|_{r=0}=-V_{d-1}\left(K \mid u^{\perp}\right), \quad u \in \mathbb{S}^{d-1},
$$

proved in [20]. Here $\partial^{+} / \partial r$ and $V_{d-1}$ stand for right derivative and $(d-1)$-dimensional volume, respectively. Theorem 6.2 from [9] is another result related to our Theorem 4, which states that most convex planar bodies are determined by the covariogram function over its entire support. 
In Section 3 we prove Theorem 3. Sections 4 and 5 are independent of each other and present proofs of Theorem 1 and Theorem 2, respectively. In Section 6 we prove Theorem 4. In this version of the paper the proofs of some of the most technical lemmas are omitted, and those of a few theorems are just sketched. The complete version is available online [3].

\section{Background from convex geometry}

The Euclidean $d$-dimensional space with origin $o$, scalar product $\langle.,$.$\rangle , and norm |\cdot|$ is denoted by $\mathbb{E}^{d}$. The unit sphere and the unit ball in $\mathbb{E}^{d}$ are denoted by $\mathbb{S}^{d-1}$ and $\mathbb{B}^{d}$, respectively. The orthogonal projection of a set $X \subseteq \mathbb{E}^{d}$ onto an affine space $L \subseteq \mathbb{E}^{d}$ is denoted by $X \mid L$. If $u \in \mathbb{E}^{d}$, then $u^{\perp}$ stands for the orthogonal complement to $u$. We denote the $j$-dimensional volume of a convex set in $\mathbb{E}^{d}$ of dimension at most $j$ by $V_{j}, j \in\{1, \ldots, d\}$. If $j=d$ then we omit the subscript and write simply $V$. We write $\mathscr{H}^{j}$ for the $j$-dimensional Hausdorff measure in $\mathbb{E}^{d}$. In what follows, in integrals on $j$-dimensional spheres in $\mathbb{E}^{d}$ (with $j \leq d-1$ ) we abbreviate $\mathscr{H}^{j}(\mathrm{~d} u)$ by $\mathrm{d} u$.

The abbreviations bd, int, relint, cl, and aff stand for boundary, interior, relative interior, closure, and affine hull, respectively.

Following the monograph [26] we denote by $\mathcal{K}^{d}$ and $\mathcal{K}_{0}^{d}$ the classes of nonempty, compact, convex sets and compact, convex sets with nonempty interior, respectively. Elements of $\mathcal{K}_{0}^{d}$ are said to be convex bodies. If $o \in K$, then $r_{K}(u):=\max \{\alpha>0: \alpha u \in K\}$, $u \in \mathbb{S}^{d-1}$, is called the radius function of $K$. We also introduce the support function of $K, h_{K}(u):=\max \{\langle x, u\rangle: x \in K\}, u \in \mathbb{E}^{d}$. The difference body of a convex body $K$ is the set $D K:=K+(-K)$. The function $w_{K}(u):=h_{D K}(u)$ is called the width function of $K$. Observe that for $u \in \mathbb{S}^{d-1}$ the quantity $w_{K}(u)$ is equal to the distance between the two distinct supporting hyperplanes of $K$ orthogonal to $u$. The face of a convex body $K \subseteq \mathbb{E}^{d}$ in the direction $u \in \mathbb{E}^{d} \backslash\{o\}$ is denoted by $F_{K}(u)$. Elements of $\mathcal{K}_{0}^{d}$ representable as intersection of a finite number of closed halfspaces are called convex $d$-polytopes. Two convex $d$-polytopes $P, Q \subseteq \mathbb{E}^{d}$ are said to be in a general relative position if, for every $u \in \mathbb{S}^{d-1}$ and $x \in \mathbb{E}^{d}$, $F_{P}(u) \cap\left(F_{Q}(u)+x\right)$ is either empty or a singleton.

If $K$ is a convex body in $\mathbb{E}^{d}$ and $u \in \mathbb{E}^{d} \backslash\{o\}$ then, for $p \in$ relint $F_{K}(u)$, the normal cone $N_{K}(p)$ does not depend on the choice of $p$ in relint $F_{K}(u)$. We denote $N_{K}(p)$ by $\bar{N}_{K}(u)$ and call it the normal cone of $K$ in direction $u$.

If $X$ is a subset of bd $K$, then the set of all outward unit normals of $K$ at points of the set $X$ is called the spherical image of $X$ with respect to $K$. Two boundary $\operatorname{arcs} A$ and $B$ of $K \subseteq \mathbb{E}^{2}$ are said to be antipodal if their spherical images with respect to $K$ are reflections of each other.

For $\varepsilon>0$ the $\varepsilon$-neighborhood of a set $X \subseteq \mathbb{E}^{d}$ is the set $X+\varepsilon \cdot$ int $\mathbb{B}^{d}$, i.e. the set consisting of all those points $x$ whose distance to some point of $X$ is strictly less than $\varepsilon$. The Hausdorff distance $\delta(X, Y)$ between nonempty compact sets $K$ and $H$ in $\mathbb{E}^{d}$ is the least possible $\alpha \geq 0$ such that $X \subseteq Y+\alpha \cdot \mathbb{B}^{d}$ and $Y \subseteq X+\alpha \cdot \mathbb{B}^{d}$. Information on the Hausdorff distance in the class of convex bodies is collected in [26, Section 1.8]. We introduce the distance function $\bar{\delta}(X, Y)$ for sets $X, Y \subseteq \mathbb{E}^{d}$ as the minimum of $\delta(X, \phi(Y))$, where $\phi$ ranges over all translations and reflections. by

The area measure of order $d-1$ of a convex body $K \subseteq \mathbb{E}^{d}$ (see [26, Section 4.2]) is given

$$
S_{d-1}(K, \omega):=\mathscr{H}^{d-1}(\{p \in \operatorname{bd} K: \text { some outward normal of } K \text { at } p \text { belongs to } \omega\}),
$$

where $\omega$ is a Borel set in $\mathbb{S}^{d-1}$. If $d=2$, then $S_{1}(K, \cdot)$ is said to be the length measure of $K$. 
Given a strictly convex body $K \subseteq \mathbb{E}^{d}, z_{K}(u), u \in \mathbb{S}^{d-1}$, denotes the boundary point of $K$ with outward normal $u$. Note that $z_{K+H}(u)=z_{K}(u)+z_{H}(u)$ for all strictly convex $K, H \subseteq \mathbb{E}^{d}$ and $u \in \mathbb{S}^{d-1}$. If $K \in \mathcal{C}_{+}^{2}$ and $d=2$, we denote the curvature of $K$ at $z_{K}(u)$ by $\tau_{K}(u)$. We parametrize the unit circle $\mathbb{S}^{1}$ in a standard manner by the vector function $u(t):=(\cos t, \sin t)$, where $t \in \mathbb{R}$, and we put $\tau_{K}(t):=\tau_{K}(u(t))$ and $z_{K}(t):=z_{K}(u(t))$. For $t_{1}, t_{2} \in \mathbb{R}$ with $t_{1} \leq t_{2}$, we introduce the notation $z_{K}\left(t_{1}, t_{2}\right):=\left\{z_{K}(t): t_{1} \leq t \leq t_{2}\right\}$.

The knowledge of $z_{K}\left(t_{1}\right)$ and the curvature $\tau_{K}(t)$ for $t \in\left[t_{1}, t_{2}\right]$ allows us to determine the $\operatorname{arc} z_{K}\left(t_{1}, t_{2}\right)$. More formally, the parametrization $z_{K}(t), t_{1} \leq t \leq t_{2}$, of $z_{K}\left(t_{1}, t_{2}\right)$ is determined from the representation

$$
z_{K}(t)=z_{K}\left(t_{1}\right)+\int_{t_{1}}^{t} \frac{u(s+\pi / 2)}{\tau_{K}(s)} \mathrm{d} s .
$$

Equality (3) can be found in [14, p. 11] and [9, p. 186] (see also a more general result [26, Theorem 4.3.2]). By (3) we see that two antipodal $\operatorname{arcs} z_{K}\left(t_{1}, t_{2}\right)$ and $z_{K}\left(t_{1}+\pi, t_{2}+\pi\right)$ with $t_{1}<t_{2}$ and $t_{2}-t_{1}<\pi$ are reflections of each other if and only if $\tau_{K}(t)=\tau_{K}(t+\pi)$ for every $t \in\left[t_{1}, t_{2}\right]$.

\section{The relationship between the local coincidence condition and covariogram data}

In this section we prove parts (i), (iii), (iv), and (v) of Theorem 3. The proof of part (ii) is postponed to Section 4 .

Sketch of the proof of Theorem 3(i). Using LC and the strict convexity of $K$, we see that for every $u \in \mathbb{S}^{d-1}$, there exists a relatively open subset $G_{u}$ of $\mathbb{S}^{d-1}$ such that $\left(-G_{u}\right) \cap G_{u}=\varnothing$, $u \in G_{u}$, and the equality

$$
\left\{z_{K}\left(G_{u}\right)+x_{1}(u),-z_{K}\left(-G_{u}\right)-x_{2}(u)\right\}=\left\{z_{H}\left(G_{u}\right),-z_{H}\left(-G_{u}\right)\right\}
$$

holds for some vector functions $x_{1}(u)$ and $x_{2}(u)$. This implies that

$$
z_{D K}\left(G_{u}\right)+x_{1}(u)-x_{2}(u)=z_{D H}\left(G_{u}\right) \quad \text { for each } u \in \mathbb{S}^{d-1} .
$$

By strict convexity there is an unique way of gluing together adjacent neighborhoods of bd $D H$ (and of bd $D K$ ) and the identity (5) implies that $D H=D K$ and, also, that $x_{1}(u)=x_{2}(u)$ for each $u \in S^{d-1}$. Once this is proved, it is straightforward to conclude, from (4), that $g_{H}$ and $g_{K}$ coincide in a neighborhood of the boundary of their common support.

For $x \in \operatorname{int} D K \backslash\{o\}$, let $p$ and $q$ be the endpoints of the $\operatorname{arc}(K+x) \cap \operatorname{bd} K$. Then $p-x$ and $q-x$ belong to $\operatorname{bd} K$ and $P_{K}(x):=\operatorname{conv}\{p, q, p-x, q-x\}$ is a parallelogram. Following [21] we define $D_{K}(x)$ to be $\pm(p-q)$ with the sign determined by the condition $\left\langle x, \mathcal{R}_{\pi / 2} D_{K}(x)\right\rangle<0$; see Figure 2. It is known that

$$
D_{K}(x)=-\mathcal{R}_{\pi / 2}\left(\nabla g_{K}(x)\right)
$$

where $\mathcal{R}_{\pi / 2}$ denotes, throughout the paper, the counterclockwise rotation about the origin by the angle $\pi / 2$ and $\nabla$ stands for the gradient. We also have the equality

$$
D_{K}\left(D_{K}(x)\right)=-x \text {. }
$$


(a)

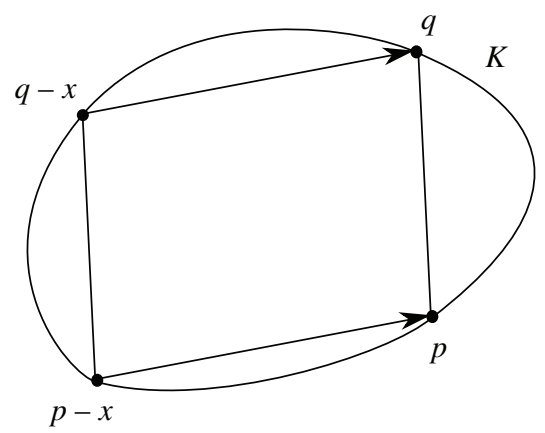

(b)

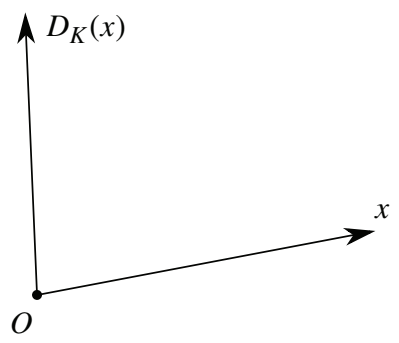

FIGURE 2: The parallelogram $P_{K}(x)$ and the vector $D_{K}(x)$.

Proof of Theorem 3(iii). First we prove that $\mathrm{GC}^{\prime}(\{o\})$ implies $\mathrm{GC}(\mathrm{bd} D K)$. In the plane $V_{1}\left(K \mid u^{\perp}\right)=w_{K}\left(\mathcal{R}_{\pi / 2} u\right)=h_{D K}(u)$ for every $u \in \mathbb{S}^{1}$. Hence, from (2) we find that $\mathrm{GC}^{\prime}(\{o\})$ implies $D K=D H$.

It is easy to prove that the mapping $D_{K}$ : int $D K \backslash\{o\} \rightarrow$ int $D K \backslash\{o\}$ is continuous and, by (7), also that its inverse is continuous. Moreover, it maps a punctured neighborhood $G$ of $o$ in a set $G^{\prime}$ with bd $D K \subseteq$ bd $G^{\prime}$. Let $G$ be a punctured neighborhood of $o$ in which $g_{K}$ and $g_{H}$ coincide, up to an additive constant. Then, in view of $(6), D_{K}(x)=-\mathcal{R}_{\pi / 2}\left(\nabla g_{K}(x)\right)=$ $-\mathcal{R}_{\pi / 2}\left(\nabla g_{H}(x)\right)=D_{H}(x)$ for all $x \in G$ and we get that $P_{K}(x)$ and $P_{H}(x)$ are translates of each other; see the related Lemma 1.5 of [6].

The set $K \backslash$ int $P_{K}(x)$ is the union of the four lunettes outside $P_{K}$ (where a lunette of $K$ is a compact set bounded by a chord of $K$ and a boundary arc of $K$ joining the endpoints of this chord). Let $p$ and $q$ be as in the definition of $D_{K}$. The sum of the areas of the two lunettes adjacent to $[p, q]$ and $[p, q]-x$ equals $g_{K}(x)$, the sum of the areas of the other two lunettes equals $g_{K}\left(D_{K}(x)\right)$. Similar considerations hold for $H$ too; thus

$$
\begin{aligned}
& g_{K}\left(D_{K}(x)\right)=V(K)-V\left(P_{K}(x)\right)-g_{K}(x)=g_{K}(o)-V\left(P_{K}(x)\right)-g_{K}(x), \\
& g_{H}\left(D_{H}(x)\right)=V(H)-V\left(P_{H}(x)\right)-g_{H}(x)=g_{H}(o)-V\left(P_{H}(x)\right)-g_{H}(x) .
\end{aligned}
$$

Since $g_{K}(x)-g_{K}(o)=g_{H}(x)-g_{H}(o)$ for $x \in G$, we obtain, from (8) and (9), $g_{K}(x)=g_{H}(x)$ for $x \in G^{\prime}$.

The proof of the converse implication is similar. An open subset $G^{\prime}$ of $D K$ with bd $G^{\prime} \supseteq$ bd $D K$ and $g_{K}(x)=g_{H}(x)$ for $x \in G$, $^{\prime}$ is mapped by $D_{K}$ onto a punctured neighborhood $\bar{G}$ of the origin. Thus, using (8) and (9), we obtain $g_{K}(x)-g_{H}(x)=V(K)-V(H)$ for $x \in G$.

Proof of Theorem 3(iv). In view of Theorem 3(i) and (ii), it suffices to construct $K$ and $H$ satisfying LC and $V(K)<V(H)$. Let $T$ be a regular triangle of unitary edge length with center at the origin. If $u_{1}, u_{2}$, and $u_{3}$ denote the unit outer normals to the edges, the length measure of $T$ is $\sum_{i=1}^{3} \delta_{u_{i}}$, where $\delta_{u_{i}}$ is the Dirac delta distribution on the manifold $\mathbb{S}^{1}$ centered in $u_{i}$. For each $i=1,2,3$, let $\phi_{i}$ be a continuous nonnegative function on $\mathbb{S}^{1}$ supported in a small arc centered at $u_{i}$, whose integral on $\mathbb{S}^{1}$ is 1 . Moreover, choose the $\phi_{i}$ in such a way that $\int_{\mathbb{S}^{1}} \sum_{i=1}^{3} \phi_{i}(u) u \mathrm{~d} u=0$. Let $K_{1}$ be a convex body in $\mathbb{E}^{2}$ whose length measure has density $\sum_{i=1}^{3} \phi_{i}$. It is clear that the measure $S_{1}\left(K_{1}, \cdot\right)$ approximates, in some sense, the measure $S_{1}(T, \cdot)$. In fact, it can be shown that the distance in the Prohorov metric (see [18] for a definition) of $S_{1}\left(K_{1}, \cdot\right)$ and $S_{1}(T, \cdot)$ can be made arbitrarily small. 
(a)

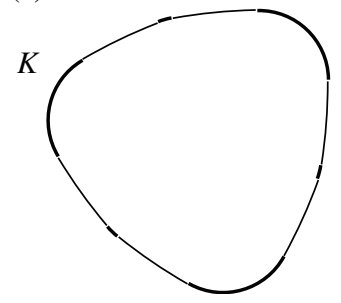

(b)

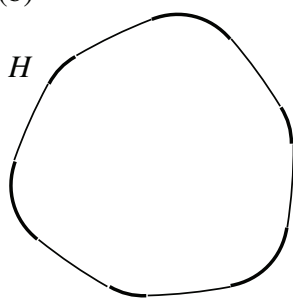

FIgURE 3: The covariograms of $H$ and $K$ coincide in a neighborhood of the boundary of their support but do not coincide in any neighborhood of $o$.

Let $K_{2}$ be a slight rotation of $K_{1}$ such that the supports of the length measures of $K_{1}$ and $K_{2}$ are disjoint. By a stability result for the Minkowski problem with respect to the Prohorov metric proved in [18, Theorem 3.1] $K_{1}+K_{2}$ is close to $2 T$, while $K_{1}-K_{2}$ is close to $D T$ in the metric $\bar{\delta}$ (Theorem 3.1 from [18] is a strengthening of Theorem 7.2.2 from [26]; see also the related Theorems 4.3.5 and 7.2.6 from this monograph). Consequently, the area of $K_{1}+K_{2}$ is close to $4 V(T)$, while the area of $K_{1}-K_{2}$ is close to $V(D T)=6 V(T)$. Let

$$
K:=K_{1}+K_{2}+\mathbb{B}^{2}, \quad H:=K_{1}-K_{2}+\mathbb{B}^{2} .
$$

See Figure 3, which depicts possible choices of bodies $K$ and $H$.

By construction, $K$ and $H$ are $\mathcal{C}_{+}^{2}$ and they satisfy LC. Thus, by Theorem 3(i) and (ii) they have equal covariograms in a neighborhood of $\operatorname{bd} D K$. Furthermore, $K$ has smaller area than $H$, since by the two-dimensional version of the Steiner formula (see [26, Section 4.1]) we have $V(K)=V\left(K_{1}+K_{2}\right)+\mathscr{H}_{1}\left(\mathrm{bd} K_{1}\right)+\mathscr{H}_{1}\left(\mathrm{bd} K_{2}\right)+\pi$ and $V(H)=V\left(K_{1}-K_{2}\right)+\mathscr{H}_{1}\left(\mathrm{bd} K_{1}\right)+$ $\mathscr{H}_{1}\left(\mathrm{bd} K_{2}\right)+\pi$.

In the proof of Theorem 3(v) we shall need the following lemma, presenting a formula which is also related to a formula given in [22, p. 18]. For the proof of Lemma 1 see [3].

Lemma 1. Let $P$ be a convex polygon in $\mathbb{E}^{2}$, and let $G(P)$ be given by

$$
G(P):=\bigcap\left\{D T: T=\operatorname{conv}\left\{p_{1}, p_{2}, p_{3}\right\}, p_{1}, p_{2}, p_{3} \text { are consecutive vertices of } P\right\} .
$$

Then $o \in \operatorname{int} G(P)$ and, for $u \in G(P)$, we have $g_{P}(u)=V(P)-w_{P}\left(\mathcal{R}_{\pi / 2} u\right)+|u|^{2} C$, where $C$ depends only on $\left\{\bar{N}_{P}\left(\mathcal{R}_{\pi / 2} u\right), \bar{N}_{P}\left(-\mathcal{R}_{\pi / 2} u\right)\right\}$.

If $d_{i} \in \mathbb{N}$ and $K_{i}$ is a convex body in $\mathbb{E}^{d_{i}}(i=1,2)$, then the covariogram function of $K:=K_{1} \times K_{2}$ is given by

$$
g_{K}(x)=g_{K_{1}}\left(x_{1}\right) g_{K_{2}}\left(x_{2}\right),
$$

where $x_{i} \in \mathbb{E}^{d_{i}}(i=1,2)$ and $x:=\left(x_{1}, x_{2}\right)$.

Proof of Theorem 3(v). We introduce convex polygons $P_{1}, P_{2} \subseteq \mathbb{E}^{2}$ which are obtained from the square $Q:=[-10,10]^{2}$ by 'cutting off' isosceles triangles at the vertices of $Q$. The polygon $P_{k}(k=1,2)$ is constructed by cutting off the isosceles triangle with lateral sides having length $\alpha_{i, j}^{k}$ at the vertex $(10 i, 10 j)$ of $Q$ for each $i, j \in\{-1,1\}$, where the constants 
(a)

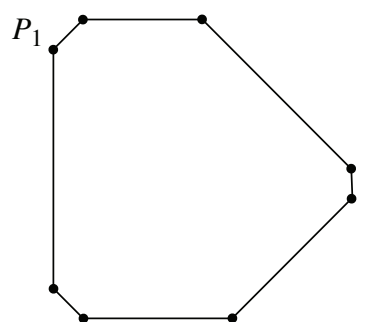

(b)

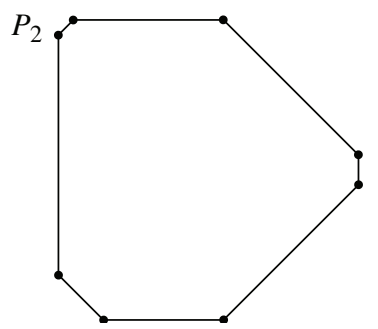

FIGURE 4: The covariograms of $P_{1}$ and $P_{2}$ have equal supports and coincide in a neighborhood of $o$, but they do not coincide in any neighborhood of the boundary of their support.

$\alpha_{i, j}^{k}$ are defined as follows:

$$
\begin{aligned}
\alpha_{1,1}^{1} & =10, & \alpha_{1,1}^{2} & =9, \\
\alpha_{-1,1}^{1} & =2, & \alpha_{-1,1}^{2} & =1, \\
\alpha_{-1,-1}^{1} & =2, & \alpha_{-1,-1}^{2} & =3, \\
\alpha_{1,-1}^{1} & =8, & \alpha_{1,-1}^{2} & =9 .
\end{aligned}
$$

See Figure 4, which depicts $P_{1}$ and $P_{2}$. No translation or reflection of $P_{1}$ coincides with $P_{2}$ and, moreover, for each $k \in\{1,2\}$,

$$
V\left(P_{k}\right)=V(Q)-\frac{1}{2} \sum_{i, j=-1}^{1}\left(\alpha_{i, j}^{k}\right)^{2}=20^{2}-\frac{1}{2} 172 .
$$

Furthermore, it is easy to see that $D P_{1}=D P_{2}$. Lemma 3.1 from [5] proves that, for each $u \in \mathbb{S}^{1}$, the knowledge of the covariogram of a convex polygon $P \subseteq \mathbb{E}^{2}$ near the boundary of its support determines the set $\left\{V_{1}\left(F_{P}(-u)\right), V_{1}\left(F_{P}(u)\right)\right\}$. But, for $u=(1,1)$, we have

$$
\{10 \sqrt{2}, 2 \sqrt{2}\}=\left\{V_{1}\left(F_{P_{1}}(-u)\right), V_{1}\left(F_{P_{1}}(u)\right)\right\} \neq\left\{V_{1}\left(F_{P_{2}}(-u)\right), V_{1}\left(F_{P_{2}}(u)\right)\right\}=\{9 \sqrt{2}, 3 \sqrt{2}\} .
$$

Hence, $g_{P_{1}}$ and $g_{P_{2}}$ do not coincide in some neighborhood of bd $D P_{1}=\operatorname{bd} D P_{2}$.

For $j \in\{1,2\}$, let $G\left(P_{j}\right)$ be defined as in the statement of Lemma 1. Since, for each $u \in \mathbb{R}^{2}$,

$$
\left\{\bar{N}_{P_{1}}\left(\mathcal{R}_{\pi / 2} u\right), \bar{N}_{P_{1}}\left(-\mathcal{R}_{\pi / 2} u\right)\right\}=\left\{\bar{N}_{P_{2}}\left(\mathcal{R}_{\pi / 2} u\right), \bar{N}_{P_{2}}\left(-\mathcal{R}_{\pi / 2} u\right)\right\},
$$

Lemma 1 implies that $g_{P_{1}}(v)=g_{P_{2}}(v)$ for each $v \in G\left(P_{1}\right) \cap G\left(P_{2}\right)$. Since this set is a neighborhood of $o$ the proof for $d=2$ is concluded by putting $K=P_{1}$ and $H=P_{2}$.

For $d \geq 3$, we define $K=P_{1} \times[-1,1]^{d-2}$ and $H=P_{2} \times[-1,1]^{d-2}$, and property (10) proves the assertion.

\section{Determination results for planar $\mathcal{C}_{+}^{2}$ bodies}

In $\left[9\right.$, Lemma 2.1] it was shown that for planar $\mathcal{C}_{+}^{2}$ convex bodies the asymptotic behaviour of the covariogram function near the boundary of its support determines the nonordered pair $\left\{\tau_{K}(u), \tau_{K}(-u)\right\}$ for every $u \in \mathbb{S}^{1}$. Thus, the following lemma holds. 


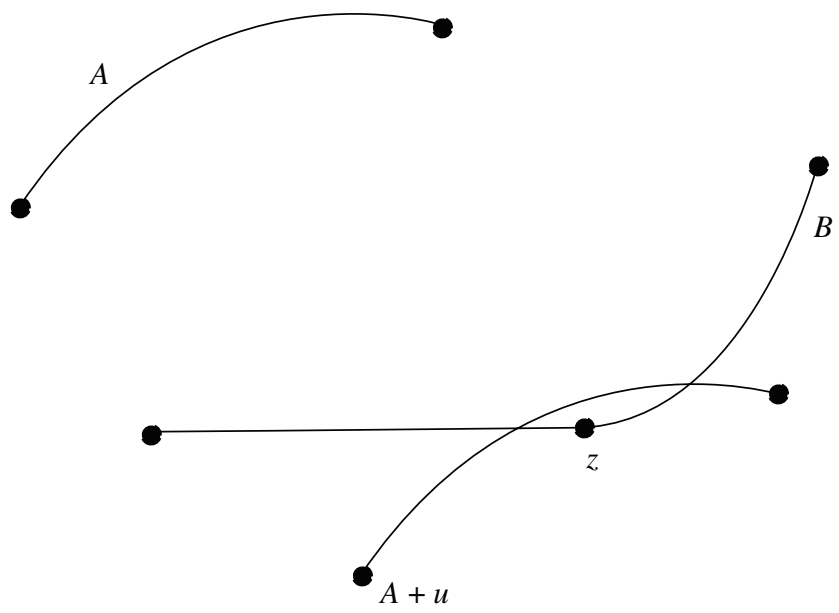

Figure 5: The $\operatorname{arc} A+u$ captures the endpoint $z$ of $B$.

Lemma 2. Let $K$ be a planar $\mathcal{C}_{+}^{2}$ convex body. Then the covariogram of $K$ over any neighborhood of bd $D K$ determines the mapping $u \mapsto\left\{\tau_{K}(u), \tau_{K}(-u)\right\}$, where $u$ ranges over $\mathbb{S}^{1}$.

Suppose that $A$ and $B$ are two disjoint antipodal boundary arcs of $K$. Let $z$ be an endpoint of $B$. Let us denote by $\bar{B}$ the convex curve obtained by joining $B$ and the appropriate half of the line which is tangent to $B$ at $z$. We say that the translated arc $A+u, u \in \mathbb{E}^{d}$, captures the endpoint $z$ of $B$ if $A+u$ intersects $\bar{B}$ at two points which bound an $\operatorname{arc}$ of $\bar{B}$ containing $z$ in its relative interior; see Figure 5.

The following lemma on capturing arcs (whose proof is given in [3]) improves slightly Lemma 4.2 of [9], since it also indicates which translation vector can be chosen for making a capture.

Lemma 3. Let $K$ be a planar $\mathcal{C}_{+}^{2}$ convex body. Assume that the antipodal arcs $z_{K}\left(t_{1}, t_{2}\right)$ and $z_{K}\left(t_{1}+\pi, t_{2}+\pi\right)$ (where $t_{1}<t_{2}$ and $t_{2}-t_{1}<\pi$ ) are not reflections of each other. Let $t^{*}$ be equal to $t_{1}$ for the case in which $\tau_{K}\left(t_{1}\right) \neq \tau_{K}\left(t_{1}+\pi\right)$ and be equal to the maximal value in $\left[t_{1}, t_{2}\right]$ such that for every $t \in\left[t_{1}, t^{*}\right]$ the equality $\tau_{K}(t)=\tau_{K}(t+\pi)$ holds, otherwise. We put $z_{0}:=\frac{1}{2}\left(z_{D K}\left(t_{1}\right)+z_{D K}\left(t^{*}\right)\right)$. Then there exists a vector $u$ arbitrarily close to $z_{0}$, such that either $z_{K}\left(t_{1}\right)$ is captured by $z_{K}\left(t_{1}+\pi, t_{2}+\pi\right)+u$ or $z_{K}\left(t_{1}+\pi\right)$ is captured by $z_{K}\left(t_{1}, t_{2}\right)-u$.

The following lemma states that $\mathcal{C}_{+}^{2}$ regularity of a planar convex body $K$ can be recognized from the covariogram of $K$ over every neighborhood of bd $D K$; see [3] for the proof.

Lemma 4. Let $K$ and $H$ be planar convex bodies with $K \in \mathcal{C}_{+}^{2}$. Then the condition $\mathrm{GC}(\mathrm{bd} D K)$ implies that $H$ also belongs to the class $\mathrm{C}_{+}^{2}$.

Proof of Theorem 1. Assume that $X=X_{0} \cup \mathrm{bd} D K$. First, by Lemma 4 we deduce that $H \in$ $\mathcal{C}_{+}^{2}$. If $K$ is centrally symmetric, then the knowledge of the mapping $u \mapsto\left\{\tau_{K}(u), \tau_{K}(-u)\right\}$, $u \in \mathbb{S}^{1}$, determines $K$. Thus, in view of Lemma 2 , we get the assertion. Now let us assume that $K$ is not centrally symmetric. Furthermore, let $X^{\prime}$ be an arbitrary open set with $X \subseteq X^{\prime}$. Let us prove, by contradiction, that $K$ is determined within the class $\mathcal{C}_{+}^{2}$ by its covariogram over $X^{\prime}$. Assume the contrary, i.e. there exists a planar convex body $H$ from the class $\mathcal{C}_{+}^{2}$ such that $H$ cannot be obtained from $K$ by reflection or translation and $g_{K}(x)=g_{H}(x)$ for all $x \in X^{\prime}$. Let $t_{0} \in \mathbb{R}$ be such that $\tau_{K}\left(t_{0}\right) \neq \tau_{K}\left(t_{0}+\pi\right)$. In [9, pp. 186-187] it was shown 
that by replacing $H$ by an appropriate translation or reflection there exist $\operatorname{arcs} A^{+}$and $A^{-}$ containing $z_{K}\left(t_{0}\right)$ and $z_{K}\left(t_{0}+\pi\right)$, respectively, in their relative interiors and contained in the set bd $K \cap$ bd $H$. Furthermore, in [9] it was also noted that if we additionally assume that $A^{+}$ and $A^{-}$are maximal arcs with the above properties, then $A^{+}$and $A^{-}$are antipodal to each other, i.e. $A^{+}=z_{K}\left(t_{1}, t_{2}\right)$ and $A^{-}=z_{K}\left(t_{1}+\pi, t_{2}+\pi\right)$, for $t_{1}, t_{2} \in \mathbb{R}$ with $t_{1}<t_{0}<t_{2}$ and $t_{2}-t_{1}<\pi$. We shall get a contradiction by showing that $A^{+}$or $A^{-}$is not maximal, i.e. there exists an arc which strictly contains $A^{+}$or $A^{-}$and is contained in $(\mathrm{bd} K) \cap(\mathrm{bd} H)$.

Obviously, $\tau_{K}(t)=\tau_{H}(t)$ for $t \in\left[t_{1}, t_{2}\right] \cup\left[t_{1}+\pi, t_{2}+\pi\right]$. By Lemma 2 we get the equality

$$
\left\{\tau_{K}(t), \tau_{K}(t+\pi)\right\}=\left\{\tau_{H}(t), \tau_{H}(t+\pi)\right\}
$$

for each $t \in \mathbb{R}$. If $\tau_{K}\left(t_{1}\right) \neq \tau_{K}\left(t_{1}+\pi\right)$, then using the equalities $\tau_{K}\left(t_{1}\right)=\tau_{H}\left(t_{1}\right), \tau_{K}\left(t_{1}+\pi\right)=$ $\tau_{H}\left(t_{1}+\pi\right),(11)$, and the continuity of the functions $\tau_{K}(t)$ and $\tau_{H}(t)$ we get that there exists an $\varepsilon>0$ such that the equality $\tau_{K}(t)=\tau_{H}(t)$ holds for $t \in\left[t_{1}-\varepsilon, t_{1}\right]$. Consequently, by (3) we have $z_{K}\left(t_{1}-\varepsilon, t_{1}\right)=z_{H}\left(t_{1}-\varepsilon, t_{1}\right)$, a contradiction to the maximality of $A^{+}$. Thus, in the sequel we assume that $\tau_{K}\left(t_{1}\right)=\tau_{K}\left(t_{1}+\pi\right)$. If there exists an $\varepsilon>0$ such that $\tau_{K}(t)=\tau_{K}(t+\pi)$ for $t \in\left[t_{1}-\varepsilon, t_{1}\right]$, then in view of (11) we have $\tau_{K}(t)=\tau_{H}(t)$ for $t \in\left[t_{1}-\varepsilon, t_{1}\right]$, which, by (3), implies the equality of the $\operatorname{arcs} z_{K}\left(t_{1}-\varepsilon, t_{1}\right)$ and $z_{H}\left(t_{1}-\varepsilon, t_{1}\right)$, a contradiction to the maximality of $A^{+}$.

Now let us switch to the case when, for every $\varepsilon>0$, the functions $\tau_{K}(t)$ and $\tau_{K}(t+\pi)$ restricted to $\left[t_{1}-\varepsilon, t_{1}\right]$ are not identically equal, i.e. there exists a $t \in\left[t_{1}-\varepsilon, t_{1}\right]$ with $\tau_{K}(t) \neq$ $\tau_{K}(t+\pi)$. Let $t^{*}$ be the maximal scalar such that $t_{1} \leq t^{*} \leq t_{2}$ and $\tau_{K}(t)=\tau_{K}(t+\pi)$ for $t \in\left[t_{1}, t^{*}\right]$. If $t^{*}=t_{1}$, we put $v=z_{K}\left(t_{1}\right)$. If $t^{*}>t_{1}$, then for some $n \in \mathcal{N}$ the $\operatorname{arc} z_{K}\left(t_{1}, t^{*}\right)$ is a translate of $\frac{1}{2} A_{n}$ or $-\frac{1}{2} A_{n}$. In this case, we put $v=x_{n}$.

By Lemma 3 we see that either the endpoint $z_{K}\left(t_{1}\right)$ of $A^{+}$can be captured by $A^{-}$or the endpoint $z_{K}\left(t_{1}+\pi\right)$ of $A^{-}$can be captured by $A^{+}$. Furthermore, the corresponding translation vector can be chosen arbitrarily close to $v$ or $-v$. Without loss of generality, we assume that $z_{K}\left(t_{1}\right)$ is captured by $A^{-}$. In [9, pp. 188-189] it was shown that in this case a small arc $z_{K}\left(t_{1}+\pi-\varepsilon^{\prime}, t_{1}+\pi\right), \varepsilon^{\prime}>0$, is determined by the knowledge of $z_{K}\left(t_{1}, t_{2}\right), z_{K}\left(t_{1}+\pi, t_{2}+\pi\right)$, and the values of the covariogram functions at points arbitrarily close to $v$. This means that we have the equality $z_{K}\left(t_{1}+\pi-\varepsilon^{\prime}, t_{1}+\pi\right)=z_{H}\left(t_{1}+\pi-\varepsilon^{\prime}, t_{1}+\pi\right)$, a contradiction to the maximality of $A^{-}$.

Theorem 3(iii) and the statement of Theorem 1 for the case in which $X=X_{0} \cup \operatorname{bd} D K$ trivially imply the statement of Theorem 1 for the case in which $X=X_{0} \cup\{o\}$.

Proof of Theorem 3(ii). We only need to verify the implication GC $\Rightarrow$ LC for planar $\mathcal{C}_{+}^{2}$ convex bodies $K$, since the reverse implication is covered by Theorem 3(i). The proof of this implication uses ideas very similar to those of the proof of Theorem 1, and therefore we omit it and direct the reader to [3] for the details.

Remark 1. It is natural to look for minimal (with respect to inclusion) sets $X$ such that $\mathrm{GC}(X)$ implies coincidence of $K$ and $H$, up to translations and reflections. Since the covariogram is $o$-symmetric, we limit our discussion to $o$-symmetric sets $X$. We claim that the set $X$ defined in Theorem 1 is minimal in the following sense. For certain $\mathcal{C}_{+}^{2}$ sets $K$ it suffices to remove from $X_{0}$ two pairs of opposite points to violate the conclusion of Theorem 1. Let us construct a corresponding counterexample. Assume that two local symmetries of $K$ have the same center of symmetry, say $o$. Let $\pm A_{1}$ and $\pm A_{2}$ be the arcs that constitute these local symmetries, and $\pm x_{1}$ and $\pm x_{2}$ be the midpoints defined as in the statement of Theorem 1, which correspond to the local symmetries $\pm A_{1}$ and $\pm A_{2}$. Let $B_{i}, i \in\{1,2,3,4\}$, be the connected components 
(in counterclockwise order) of bd $K \backslash\left( \pm A_{1} \cup \pm A_{2}\right)$. We claim that there exist a convex body $H$ which is not a translation or a reflection of $K$ and such that $\operatorname{GC}\left(X \backslash\left\{ \pm x_{1}, \pm x_{2}\right\}\right)$ holds. It suffices to define $H$ as the body obtained from $K$ by flipping the boundary $\operatorname{arcs} B_{1}$ and $B_{3}$. That is, the boundary of $H$ is composed of the $\operatorname{arcs} \pm A_{i}(i=1,2), B_{2}, B_{4},-B_{1}$, and $-B_{3}$. The bodies $K$ and $H$ satisfy LC and thus their covariograms coincide in a neighborhood of bd $D K \cup\{o\}$, by Theorem 3(iii). Moreover, if $C$ and $D$ are the arcs which constitute a local symmetry of $K$, different from $\pm A_{1}$ and $\pm A_{2}$, then the boundaries of $K$ and $H$ (properly translated and, possibly, reflected) coincide in a neighborhood of $C$ and $D$. Therefore, $g_{K}$ and $g_{H}$ coincide in a neighborhood of the midpoints corresponding to the local symmetry. We emphasize that the example constructed here is similar in nature to the example from the proof of Theorem 4 (for the case in which $d=2$ ).

\section{Determination results for symmetric and locally symmetric bodies}

Lemma 5. let $K$ and $H$ be strictly convex bodies in $\mathbb{E}^{2}$. Let $K$ be o-symmetric and let $D K=$ $D H$. Then, for scalars $t_{0}, t_{1}$, and $t_{2}$ with $t_{0} \leq t_{1} \leq t_{2}$ and $t_{2}-t_{0} \leq \pi$, the subset

$$
z_{H}\left(t_{1}, t_{2}\right) \cup z_{H}\left(t_{1}+\pi, t_{2}+\pi\right) \cup\left\{z_{H}\left(t_{0}\right), z_{H}\left(t_{0}+\pi\right)\right\}
$$

of bd $H$ is centrally symmetric if and only iffor every $t \in\left[t_{1}, t_{2}\right]$ we have $\nabla g_{K}\left(x_{t}\right)=\nabla g_{H}\left(x_{t}\right)$, where $x_{t}:=\frac{1}{2}\left(z_{D K}\left(t_{0}\right)+z_{D K}(t)\right)$.

Proof. Let us prove the sufficiency. The equality $\nabla g_{K}\left(x_{t}\right)=\nabla g_{H}\left(x_{t}\right)$ is equivalent to the condition that $P_{H}\left(x_{t}\right)$ is a translate of $P_{K}\left(x_{t}\right)$ (see (6) for the relation among the gradient and $\left.P_{K}\right)$. But, since $K$ is $o$-symmetric, $P_{K}\left(x_{t}\right)=\operatorname{conv}\left\{ \pm \frac{1}{2} z_{D K}\left(t_{0}\right), \pm \frac{1}{2} z_{D K}(t)\right\}$. Consequently, the diagonals of $P_{H}\left(x_{t}\right)$ are translates of $\left[o, z_{D K}\left(t_{0}\right)\right]$ and $\left[o, z_{D K}(t)\right]$. The chord $\left[z_{H}\left(t_{0}\right), z_{H}\left(t_{0}+\pi\right)\right]$ is the only chord of $H$ being a translate of $\left[o, z_{H}\left(t_{0}\right)-z_{H}\left(t_{0}+\pi\right)\right]$ (because that chord is an affine diameter, i.e. $z_{H}\left(t_{0}\right)$ and $z_{H}\left(t_{0}+\pi\right)$ are antipodal). Since

$$
z_{H}\left(t_{0}\right)-z_{H}\left(t_{0}+\pi\right)=z_{D H}\left(t_{0}\right)=z_{D K}\left(t_{0}\right),
$$

$\left[z_{H}\left(t_{0}\right), z_{H}\left(t_{0}+\pi\right)\right]$ is a diagonal of $P_{H}\left(x_{t}\right)$. A similar argument implies that $\left[z_{H}(t), z_{H}(t+\pi)\right]$ is the other diagonal of $P_{H}\left(x_{t}\right)$. Hence, for every $t \in\left[t_{1}, t_{2}\right]$, the point $z_{H}(t)$ is a reflection of $z_{H}(t+\pi)$ with respect to the midpoint $\frac{1}{2}\left(z_{H}\left(t_{0}\right)+z_{H}\left(t_{0}+\pi\right)\right)$ of the diagonal $\left[z_{H}\left(t_{0}\right), z_{H}\left(t_{0}+\right.\right.$ $\pi)]$ and the sufficiency is verified.

Now let us show the necessity. If $z_{H}\left(t_{1}, t_{2}\right) \cup z_{H}\left(t_{1}+\pi, t_{2}+\pi\right) \cup\left\{z_{H}\left(t_{0}\right), z_{H}\left(t_{0}+\pi\right)\right\}$ is centrally symmetric, then a translate of this set is contained in $\frac{1}{2} \mathrm{bd} D H$. Since $D K=D H$ and $K$ is $o$-symmetric, a translate of the same set coincides with $z_{K}\left(t_{1}, t_{2}\right) \cup z_{K}\left(t_{1}+\pi, t_{2}+\pi\right) \cup$ $\left\{z_{K}\left(t_{0}\right), z_{K}\left(t_{0}+\pi\right)\right\}$. The latter implies that for every $t \in\left[t_{1}, t_{2}\right]$ the parallelogram $P_{H}\left(x_{t}\right)$ is a translate of $P_{K}\left(x_{t}\right)$ and, in view of (6), shows the sufficiency.

Proof of Theorem 2(i). The equality $D K=D H$ implies that $H$ is strictly convex. Let $G$ be any open set containing $A$. We pick an arbitrary $s \in \mathbb{R}$ and show that for a sufficiently small $\varepsilon>0$ the boundary $\operatorname{arcs} \operatorname{bd} H \cap\left(z_{H}(s)+\varepsilon \cdot \mathbb{B}^{2}\right)$ and bd $H \cap\left(z_{H}(s+\pi)+\varepsilon \cdot \mathbb{B}^{2}\right)$ around the antipodal points $z_{H}(s)$ and $z_{H}(s+\pi)$, respectively, are symmetric with respect to a reflection in a point. If $t$ is ranging from $s$ to $s+\pi$, then the midpoint of the chord $\left[z_{D H}(s), z_{D H}(t)\right]$ of $D H$ traverses a path starting at $z_{D K}(s)$ and terminating at the origin. Thus, for some $t_{0} \in[s, s+\pi]$, the midpoint $\frac{1}{2}\left(z_{D H}(s)+z_{D H}\left(t_{0}\right)\right)$ of $\left[z_{D H}(s), z_{D H}\left(t_{0}\right)\right]$ lies in $A$; see Figure 6 .

Clearly, for some $\varepsilon>0$, the midpoint of $\left[z, z_{D H}\left(t_{0}\right)\right]$ lies in $G$ for all $z \in \operatorname{bd} D H$ with $\left|z-z_{D H}(s)\right|<\varepsilon$. Let $t_{1}$ and $t_{2}$ be scalars such that

$$
\operatorname{bd} D H \cap\left(z_{D H}(s)+\varepsilon \cdot \mathbb{B}^{2}\right)=\left\{z_{D H}(t): t_{1} \leq t \leq t_{2}\right\} .
$$




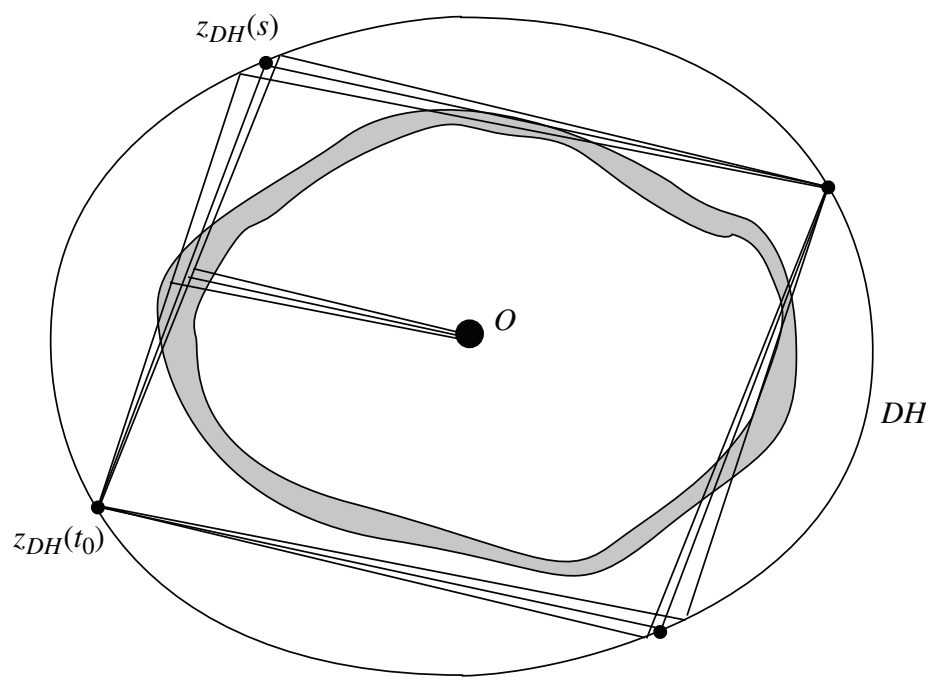

Figure 6: The set $G$ containing $A$ is shaded in gray

The assumption of the theorem and (6) imply that $\nabla g_{H}\left(x_{t}\right)=\nabla g_{K}\left(x_{t}\right)$, where $x_{t}:=\frac{1}{2}\left(z_{D H}\left(t_{0}\right)\right.$ $\left.+z_{D H}(t)\right)$ and $t \in\left[t_{1}, t_{2}\right]$. Hence, in view of Lemma $5, z_{H}\left(t_{1}, t_{2}\right)$ is a reflection of $z_{K}\left(t_{1}+\right.$ $\left.\pi, t_{2}+\pi\right)$ in a point. Thus, each pair of antipodal points of $H$ can be enclosed in the relative interior of symmetric boundary arcs of $H$, which implies the central symmetry of $H$.

Proof of Theorem 2(ii). The case in which $X=\{o\}$ can be transformed to the case in which $X=\operatorname{bd} D K$ using Theorem 3(iii). Thus, we assume that $X=\operatorname{bd} D K$. The statement of the theorem then follows from Lemma 5 applied for arbitrary $t_{0} \leq t_{1} \leq t_{2}$ with $t_{0}=t_{1}$ and $t_{2}$ sufficiently close to $t_{1}$.

Proof of Corollary 2. Let $K$ be an arbitrary locally symmetric convex body in $\mathbb{E}^{2}$. Let us show that $g_{K}$ determines $K$, up to translations and reflections. We assume that $K$ is strictly convex, since for nonstrictly convex bodies the determination was verified in [6, Theorem 1.1]. By Theorem 2(ii) we can determine all local symmetries of $K$, up to translations. But then the theorem follows from Proposition 1.4 of [6], stating that if additionally to the knowledge of $g_{K}$ a nondegenerate boundary arc of $K$ is known, then $K$ can be determined uniquely, up to translations and reflections.

\section{Genericity results}

The Nikodym distance $\delta_{N}$ (also known as the symmetric difference metric) between convex bodies $K$ and $H$ in $\mathbb{E}^{d}$ is given by $\delta_{N}(K, H)=V((K \backslash H) \cup(H \backslash K))$. It is known that $\delta_{N}$ generates the same topology in the class of convex bodies as the Hausdorff distance $\delta$ [26, pp. 58-59]. Furthermore, the inequality (see [9, p. 195])

$$
\left|g_{K}(x)-g_{H}(x)\right| \leq 2 \delta_{N}(K, H)
$$

for all $x \in \mathbb{E}^{d}$, shows that the operator $K \mapsto g_{K}$ is continuous provided that the class of convex bodies is endowed with the Nikodym distance, and the distance between covariograms is measured with respect to the maximum norm. 
The proof of the following lemma is given in [3].

Lemma 6. The class of totally nonsymmetric $\mathcal{C}_{+}^{2}$ planar convex bodies is dense in the class of all $\mathrm{C}_{+}^{2}$ planar convex bodies, with respect to the Hausdorff metric.

Now we are ready to give the proof of the genericity statement given in Theorem 4(i). We shall settle the cases in which $d=2$ and $d \geq 3$ independently of each other.

Sketch of the proof of Theorem 4 for $d=2$. Let $\mathcal{K}^{\prime}$ be the class of all planar convex bodies which are not determined, up to translations and reflections, by the covariogram over every neighborhood of bd $D K$. It can be proved (see [3] for details) that $\mathcal{K}^{\prime}$ can be decomposed as a countable union of closed sets. This implies that the complement $\mathcal{U}$ of $\mathcal{K}^{\prime}$ is a countable intersection of open sets. Using Corollary $1(i)$, we get that $U$ contains all $C_{+}^{2}$ totally nonsymmetric planar convex bodies. Furthermore, applying Lemma 6, we see that $\mathcal{U}$ is dense in the class of all convex bodies in $\mathbb{E}^{2}$. This proves part (i). The proof of part (ii) follows from similar ideas and Theorem 3(iii).

Sketch of the proof of Theorem 4(i) for $d \geq 3$. As was mentioned in [16], it is sufficient to prove the determination property for $g_{P}(x)$ in the case in which $P$ is a simplicial polytope such that $P$ and $-P$ are in general relative position. It is known (see [16, p. 86] and [27, Theorem 2.1]) that if a polytope $H$ has the difference body $D P$ (for $P$ as above), then $H=(1-\lambda) P+\lambda(-P$ ) for some $\lambda \in[0,1]$. Thus, clearly for the determination of $H$, up to translations and reflections, it is sufficient to retrieve the set $\{1-\lambda, \lambda\}$. This set can be determined using the asymptotic behaviour of $g_{P}$ near the facets of its support. See [3] for the details.

Given a convex body $K \in \mathcal{K}_{0}^{d}$ and a vector $x \in \mathbb{E}^{d}$, we introduce the body

$$
K(x):=\left\{y \in K: V_{1}(K \cap \operatorname{aff}\{y, y+x\}) \geq|x|\right\},
$$

which is the union of all those chords of $K$ that are parallel to $x$ and are not shorter than $[o, x]$. It can easily be shown that $K \cap(K+x)=K(x) \cap(K(x)+x)$. Consequently,

$$
g_{K(x)}(x)=g_{K}(x) .
$$

Let

$$
\bar{K}(x):=\left\{y \in x^{\perp}: V_{1}(K \cap \operatorname{aff}\{y, y+x\}) \geq|x|\right\} .
$$

Clearly, $\bar{K}(x)$ is the orthogonal projection of $K(x)$ onto $x^{\perp}$. Therefore, by (2) we obtain

$$
\frac{\partial}{\partial t} g_{K}(t u)=-V_{d-1}(\bar{K}(t u))
$$

Now let us consider the next proof.

Proof of Theorem 4 (counterexample). The counterexample constructed below is strongly related to a counterexample from [16]. Let $U_{1}$ and $U_{2}$ be relatively open subsets of $\mathbb{S}^{d-1}$ bounded by $(d-2)$-dimensional spheres and such that the sets $\pm U_{1}$ and $\pm U_{2}$ are mutually disjoint. Let $K$ be a $\mathcal{C}_{+}^{2}$ convex body satisfying the conditions $r_{K}(u)=1$ for $u \in \mathbb{S}^{d-1} \backslash\left(U_{1} \cup U_{2}\right)$ and $r_{K}(u)<1$ for $u \in\left(U_{1} \cup U_{2}\right)$. Then we introduce the body $H$ defined by $r_{H}(u):=r_{K}(-u)$ for $u \in\left(-U_{1}\right) \cup U_{1}$ and $r_{H}(u):=r_{K}(u)$, otherwise; see Figure 7 for an illustration of the case in which $d=2$. It can be seen that $D K=D H$.

Let

$$
A_{1}:=\left\{z_{K}(u): u \in \mathbb{S}^{d-1} \backslash\left(U_{1} \cup\left(-U_{1}\right)\right)\right\}, \quad A_{2}:=\left\{z_{K}(u): u \in \mathbb{S}^{d-1} \backslash\left(U_{2} \cup\left(-U_{2}\right)\right)\right\} .
$$



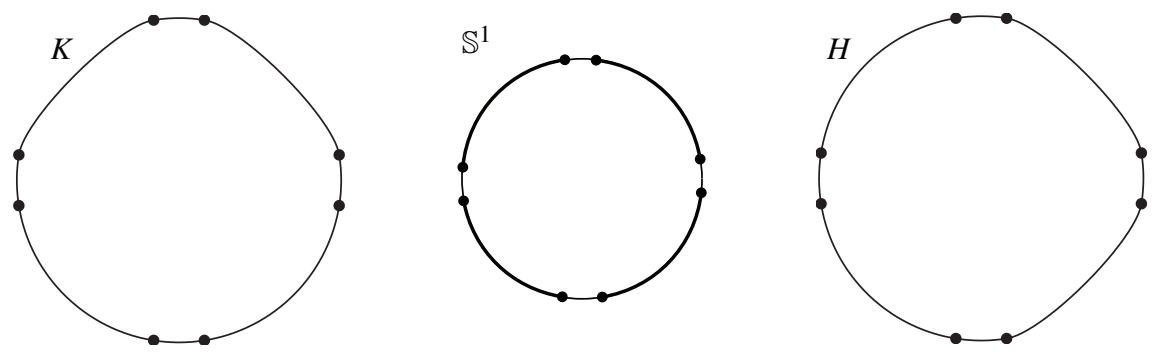

Figure 7: The bodies $K$ and $H$ satisfy GC(bd $D K \cup\{o\})$ and are not translations or reflections of each other.

Since bd $K=A_{1} \cup A_{2}$ and $\mathrm{bd} H=\left(-A_{1}\right) \cup A_{2}$, the bodies $K$ and $H$ satisfy LC. Thus, by Theorem 3(i) and (ii), $g_{K}=g_{H}$ in a neighborhood of the boundary of their support.

By standard compactness arguments, there exists an $\alpha>0$ such that, for every $x \in \mathbb{E}^{d}$ with $|x|<\alpha$ and for every two-dimensional linear space $L$ containing $x$, the endpoints of the two chords of $K \cap L$ which are translates of $[o, x]$ either all belong to $A_{1}$ or all belong to $A_{2}$. Hence, for every $u, v \in \mathbb{S}^{d-1}$ with $\langle v, u\rangle=0$ and for $|t|<\alpha$, we have

$$
\left\{r_{\bar{K}(t u)}(v), r_{\bar{K}(t u)}(-v)\right\}=\left\{r_{\bar{H}(t u)}(v), r_{\bar{H}(t u)}(-v)\right\}
$$

We recall that the volume of a convex body $K$ in $\mathbb{E}^{d}$ with $o \in K$ can be written as

$$
V(K)=\frac{1}{d} \int_{\mathbb{S}^{d-1}} r_{K}(u)^{d} \mathrm{~d} u .
$$

Let $u \in \mathbb{S}^{d-1}$ and $t$ be such that $0<t<\alpha$ and $g_{K}(t u)>0$. Up to translations of $K$, we may assume that $o \in \bar{K}(t u)$, and we have

$$
\begin{aligned}
\frac{\partial}{\partial t} g_{K}(t u) & =-V_{d-1}(\bar{K}(t u)) \quad(\text { by }(12)) \\
& =-\frac{1}{d-1} \int_{\mathbb{S}^{d-1} \cap u^{\perp}} r_{\bar{K}(t u)}(v)^{d-1} \mathrm{~d} v \quad(\text { by }(14)) \\
& =-\frac{1}{2(d-1)} \int_{\mathbb{S}^{d-1} \cap u^{\perp}}\left(r_{\bar{K}(t u)}(v)^{d-1}+r_{\bar{K}(t u)}(-v)^{d-1}\right) \mathrm{d} v \\
& =\frac{\partial}{\partial t} g_{H}(t u) \quad(\text { by }(13)) .
\end{aligned}
$$

In view of the equalities, $V(K)=g_{K}(o)=g_{H}(o)=V(H)$, the latter implying the coincidence of $g_{K}$ and $g_{H}$ for $x \in \mathbb{E}^{d}$ in a neighborhood of $o$.

\section{Acknowledgements}

Research supported by the Marie Curie Research Training Network (project Phenomena in High Dimensions, contract number MRTN-CT-2004-511953) and by Deutsche Forschungsgemeinschaft (project $A V 85 / 1-1$ ). 


\section{References}

[1] Adler, R. J. and Pyke, R. (1991). Problem 91-3. Inst. Math. Statist. Bull. 20, 409.

[2] Adler, R. J. And Pyke, R. (1997). Scanning Brownian processes. Adv. Appl. Prob. 29, 295-326.

[3] Averkov, G. And Bianchi, G. (2007). Retrieving convex bodies from restricted covariogram functions. Preprint. Available at http://www.arxiv.org/abs/math/0702892.

[4] BaAKe, M. and Grimm, U. (2007). Homometric model sets and window covariograms. Z. Kristallographie 222, 54-58.

[5] Bianchi, G. (2002). Determining convex polygons from their covariograms. Adv. Appl. Prob. 34, 261-266.

[6] Bianchi, G. (2005). Matheron's conjecture for the covariogram problem. J. London Math. Soc. (2) 71, $203-220$.

[7] Bianchi, G. (2005). Some open problems regarding the determination of a set from its covariogram. Le Matematiche (Catania) 40, 247-257.

[8] Bianchi, G. (2006). The covariogram determines three-dimensional convex polytopes. Preprint.

[9] Bianchi, G., Segala, F. And Volčič, A. (2002). The solution of the covariogram problem for plane $\mathcal{C}_{+}^{2}$ convex bodies. J. Differential Geometry 60, 177-198.

[10] CABo, A. AND BADDEley, A. (2003). Estimation of mean particle volume using the set covariance function. Adv. Appl. Prob. 35, 27-46.

[11] Enns, E. G. And Ehlers, P. F. (1978). Random paths through a convex region. J. Appl. Prob. 15, $144-152$.

[12] EnNs, E. G. AND Ehlers, P. F. (1988). Chords through a convex body generated from within an embedded body. J. Appl. Prob. 25, 700-707.

[13] Enns, E. G. And Ehlers, P. F. (1993). Notes on random chords in convex bodies. J. Appl. Prob. 30, 889-897.

[14] Gage, M. and Hamilton, R. S. (1986). The heat equation shrinking convex plane curves. J. Differential Geometry 23, 69-96.

[15] Gardner, R. J. (1995). Geometric Tomography. (Encyclopaedia Math. Appl. 58). Cambridge University Press.

[16] Goodey, P., Schneider, R. ANd WeIL, W. (1997). On the determination of convex bodies by projection functions. Bull. London Math. Soc. 29, 82-88.

[17] Gruber, P. M. (1993). Baire categories in convexity. In Handbook of Convex Geometry, Vol. A, B, North-Holland, Amsterdam, pp. 1327-1346.

[18] Hug, D. And Schneider, R. (2002). Stability results involving surface area measures of convex bodies. Rend. Circ. Mat. Palermo (2) Suppl. 21-51.

[19] Mallows, C. L. and Clark, J. M. C. (1970). Linear-intercept distributions do not characterize plane sets. J. Appl. Prob. 7, 240-244.

[20] Matheron, G. (1975). Random Sets and Integral Geometry. John Wiley, New York.

[21] Matheron, G. (1986). Le covariogramme géometrique des compacts convexes des $\mathbb{R}^{2}$. Tech. Rep. 2/86, Centre de Géostatistique, Ecole des Mines de Paris.

[22] NAGEL, W. (1991). Das geometrische Kovariogramm and verwandte Größen zweiter Ordnung. Habilitationsschrift, Friedrich-Schiller-Universität Jena.

[23] Nagel, W. (1993). Orientation-dependent chord length distributions characterize convex polygons. J. Appl. Prob. 30, 730-736.

[24] Santaló, L. A. (2004). Integral Geometry and Geometric Probability, 2nd edn. Cambridge University Press.

[25] Schmitt, M. (1993). On two inverse problems in mathematical morphology. In Mathematical Morphology in Image Processing (Opt. Eng. 34), Dekker, New York, pp. 151-169.

[26] Schneider, R. (1993). Convex Bodies: The Brunn-Minkowski Theory (Encyclopaedia Math. Appl. 44). Cambridge University Press.

[27] Schneider, R. (1994). Polytopes and Brunn-Minkowski theory. In Polytopes: Abstract, Convex and Computational, Kluwer, Dordrecht, pp. 273-299.

[28] SchneIder, R. (1998). On the determination of convex bodies by projection and girth functions. Results Math. 33, 155-160.

[29] Serra, J. (1984). Image Analysis and Mathematical Morphology. Academic Press, London.

[30] Skiena, S. S. (2004). Geometric reconstruction problems. In Handbook of Discrete and Computational Geometry, 2nd edn, Boca Raton, FL, pp. 665-676.

\section{Added in proof}

The authors of this paper have recently confirmed the validity of Matheron's conjecture for general convex bodies in the plane. This result is proved in

Averkov, G. AND Bianchi, G. (2007). Confirmation of Matheron's conjecture on covariogram of planar convex bodies. Preprint. 